valve in the aortic area may produce a systolic murmur in the aortic area. The diagnosis made difficult unless there is an associated stenosis producing a thrill and the usual pulse features. It is important because it is sometimes associated with the superposition of subacute bacterial endocarditis. The systolic murmurs described by Austin Flint and Grahame Steele have already been mentioned, and it is important to $r \in m \in m b e r$ that they occur very frequently. The systolic murmur in the mitral area, appearing for the first time in late adult life, may be the first sign of aortic reflux from syphilitic aortitis, and it will require very careful auscultation to hear the early diastolic whiff in the midsternal region. In children especially, severe exercise or increased cardiac output from any cause, but particularly that associated with fevers, may produce a soft, blowing systolic murmur at the mitral area, and this finding often leads to unnecessary worry by both the doctor and the patient's parents. It is only discovered whilst the cardiac rate is accelerated, and is very soft and blowing in nature. The resumption of the normal cardiac rhythm is soon followed by the disappearance of this murmur. In pericarditis, especially when of slight degree, a soft murmur, systolic in time, may be found over some part of the precordia, but careful auscultation will disclose its nature in most cases, and it may be followed by a very short diastolic murmur of similar character, and these sounds may be intensified by pressure with the stethoscope. Characteristically, the sounds can be described by the words "frou-frou." Exocardial murmurs are often systolic in time, as are the cardio-respiratory murmurs. Many of them can be made to disappear by instructing the patient to hold his breath, either in the position of maximum inspiration or maximum expiration.

A haemic murmur associated with a severe anaemic may appear at any part of the precordia. They are soft murmurs, which are markedly influenced by the position of the patient, often being audible only when the patient is lying down, or being intensified in that position. These murmurs are always systolic in time, often not starting until half-way through systole and always ending before the second sound appears. But a haemic murmur must never be diagnosed unless there is evidence of a severe anaemia.

From consideration of these points it will be seen that it is usually possible to make the $\sigma$ diagnosis of the cause of a systolic murmur much more concrete than was at one time considered $\delta$ possible by clinical methods alone. One has not attempted to cover the whole range of possibles diagnosis which might be attempted by cardiologists, but it is hoped that such points as have been made will help to stimulate more exact diagnosis of the causes of systolic murmurs and to $\$$ place them in their correct perspective in relation to prognosis.

A. RAE GILCHRIST, Edinburgh Post-Graduate Lectures, 1940-4I, 2, 70.

\title{
Clinical Page
}

\section{THREE CASES ILLUSTRATING RARE DISEASES OF THE ILEO-CAECAL REGION}

\author{
By A. E. MORTIMER WOOLF, M.B., B.Ch.(CAntaB), F.R.C.S. \\ (Senior Surgeon Queen Mary's Hospital for East End; Surgeon to the E.M.S., etc.).
}

Ileo-caecal tuberculosis is a rare disease. Of recent years its incidence has diminished, probably because many cases in the past have been wrongly classified. Since Crohn described the condition of terminal ileitis, it has become apparent that this condition (the causal agent of which is quite unknown) embraced many cases erroneously thought to be tuberculosis. The following experience is an example of this error.

In I924, I was consulted by a man of $3 I$ with a lump in the right iliac fossa. This had been diagnosed as appendicitis and an operation had been performed at a London hospital. The man was having indigestion, pain, probably of an obstructive character, and was losing weight. He looked ill, and there was a small healed scar about one inch long just inside the antero-superior spine. A mass could be felt in the right iliac fossa. I diagnosed ileo-caecal tuberculosis. At the time I was unable to prove or disprove this, because the patient made me promise that if he consented to operation, I was to remove no part of his anatomy. $\mathrm{He}$ 
refused to ${ }^{r}$ consent to operation unless I made this promise. I therefore opened his abdomen, and found a large mass involving the caecum and terminal end of the ileum, which at that time I had no doubt was what was then called ileo-caecal tuberculosis. I performed a lateral anatomosis between the ileum and ascending colon. For five years the patient remained well, and the mass in the right iliac fossa gradually disappeared, until merely an ill-defined, thickening was all that could be felt. Six years after the previous operation he began to complain of diarrhoea and rapidly went down hill. Indeed, in a few weeks time, it looked as if he were going to die. As a measure of desperation, I performed caecostomy and washed out his intestine. Improvement almost immediately took place, and eventually he was discharged from hospital. The caecostomy then was healed. He remained well for another five years, put on weight and carried on with his work, but he had occasional attacks of abdominal pain, which were relieved by discharge of gas and intestinal content through the caecostomy, which opened spontaneously from time to time. These attacks became more frequent and eventually, as soon as his caecostomy healed, pain would occur. He begged me to do something for him. That matters were urgent is shown by the fact that he now withdrew his embargo on my removing a portion of his intestine. I reopened the abdomen, and found a condition typical of Crohn's disease. The ileum, from the ileo-caecal junction upwards for $I_{2} \frac{1}{2}$ feet, was the replica of a piece of hose-pipe. The caecum was an indistinct mass, matted up and confused with the loop formed by the original anastomosis. The ileum was divided clear. of the disease and this, together with the ascending colon and a portion of the transverse colon, was removed. Restoration of the bowel was made by end-to-end anastomosis of the ileum to the transverse colon. An uninterrupted recovery took place, and the patient was discharged perfectly well.

The specimen showed an extensive submucous thickening of an inflammatory nature. The disease was most marked in the ileum, whose lumen was reduced to the size of a filiform urethral bougie by the extreme thickening of the intestinal wall, chiefly of the sub-mucous coat. The caecum was affected to only a minor degree, but the ileo-caecal valve was markedly thickened and prolapsed into the caecum. To my distress, it was apparent that, despite my belief that I had resected high enough to be clear of the disease, such was not the case. He remained well for eight years more, when pain and diarrheoa again occurred. At first I advised against further operation; eventually, at his own earnest request, I reopened his abdomen and found a recurrence. A further resection this time proved fatal.

No causal organism was found, nor was any evidence of tuberculosis ever obtained. The specimen was in the Hunterian Museum of The Royal College of Surgeons, but has now probably been destroyed.

Here, then was a prolonged infection of the terminal ileum observed by me over a period of I6 years, causing thickening of the intestinal wall, leading to stenosis and limited to the small intestine. Compare this with the following case of true tuberculosis of the ileocaecal region.

In October I94I, I operated on a gentleman, aet. 33, for a left inguinal hernia. Apart from this, he had been feeling run down and had definitely been over-working. He had been overhauled by a well-known physician, who reported that his chest was quite clear-afterwards confirmed by X-ray-and that he could find no evidence of disease. Examination of the abdomen revealed no abnormality, apart from the left inguinal hernia. A radical cure was performed, and recovery was uneventful. Five months later, in February 1942, he began to have abdominal pain. A barium enema now showed a marked filling defect (see Fig. I) in the inner wall of the caecum and when I was asked to see him again, a definite mass could be felt in the right iliac fossa. This had evidently developed in the time that had elapsed since his hernia operation. The diagnosis was not very certain; it rested between carcinoma of the caecum and an inflammatory condition, possibly Crohn's disease, possibly an appendix abscess, though this latter was not very likely. His temperature was up to $100^{\circ}$ at night, and the mass was tender.

Laparotomy revealed a mass in the caecum, but the terminal ileum was not thickened, though the peritoneal coat was infiltrated. The thickening in the caecum had not the hardness of carcinoma, and the peritoneal coat was studded with small infiltrated masses, which seemed not unlike conglomerate tubercles. On the whole, I thought the condition was true tuberculosis. There was no evidence of the disease elsewhere in the abdomen, apart from one or two enlarged mesenteric glands at the ileo-caecal angle. Resection of about Io inches of 


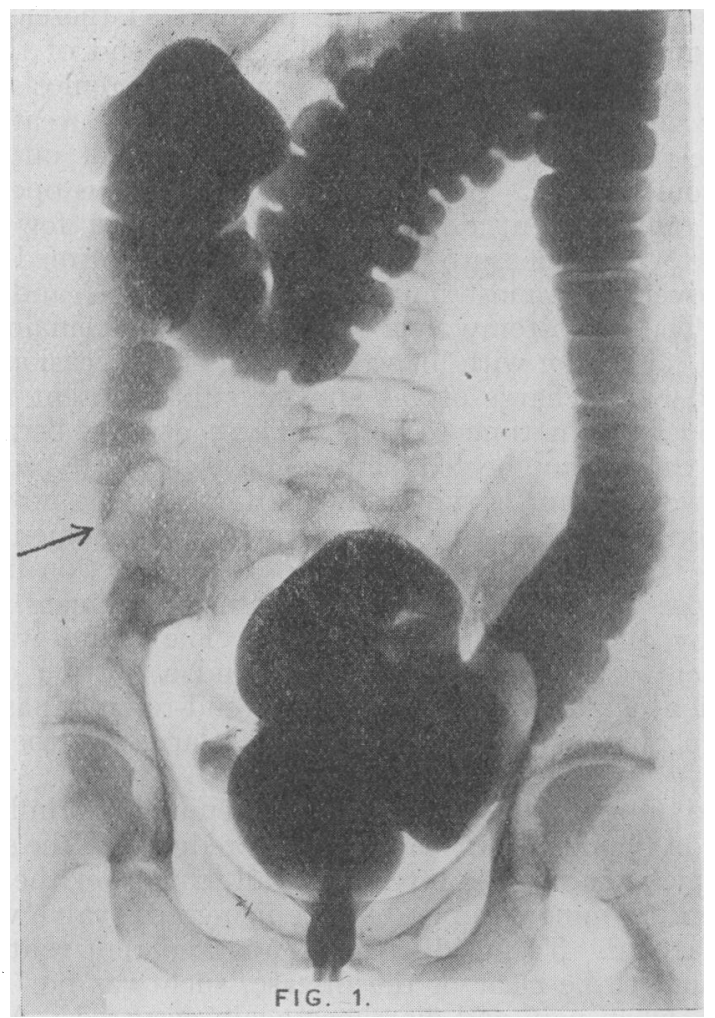

terminal ileum and right hemi-colectomy was performed, and the ileum joined to the transverse colon by end-to-end anastomosis. Recovery was uneventful, and the patient was discharged four weeks after the operation.

The specimen showed an inflammatory infiltration of the sub-mucous coats. A microscopical section gave the typical appearance of tuberculosis. Tubercles were also seen on the peritoneal surface of the ileum and extended upwards on the ascending colon for a short distance. The greater portion of the disease was in the caecum, from which situation it had evidently spread in both directions.. A few mesenteric glands also showed the typical appearance of tuberculosis.

In this case, the condition had apparently developed in a relatively short period, certainly in less than six months. Of course it is impossible to say that at the time of the hernia operation there was not some slight disease in the caecum, as exploration here was not performed. The events which ultimately led up to operation were definitely speedy, as exemplified by the formation of the tumour, which was not felt by palpation at the time of his X-ray examination. It is true that, generally speaking, tuberculosis is a slow disease, and though of course there are a number of instances in various parts of the body where it is known that it may develop acutely, yet, on the whole, one is not very tempted to diagnose tuberculosis on a short history. But Crohn's disease is definitely a condition of long duration, and the first case quoted above was watched for as long as I5 years, and is a very good example of this.

It is interesting, even if unprofitable, to reflect on some of the etiological factors of these two conditions, and the following questions spring to one's mind. Why should tuberculosis, which is not an uncommon disease in the abdomen, in certain rare cases confine itself to the ileo-caecal angle, and this apparently without widespread affection of the rest of the contents of the abdomen? Why should Crohn's disease, or terminal ileitis, affect the lower end of the ileum and stop short at the caecum, and yet, although this is true of the majority of cases, it is by no means invariable? Crohn's disease has been seen in the jejunum, but this is rare. On the other hand, although the tubercle bacillus is a well-known entity, yet the causal organism of Crohn's disease is quite unknown. We do not know if it is a virus or if it is some organism 
not yet discovered; and yet in an organism like tubercle, a specificity apparently sometimes exists whereby the caecum and its immediate surroundings alone are attacked. It would almost look as if there were varying conditions in the intestinal efflux which render the walls of the intestine susceptible to definite specific diseases. This seems to be the case with ulcerative colitis, the causal agent of which has never been discovered, but which seems to depend on the intestinal contents which are poured out through the ileo-caecal valve. Hence the value of the operation of ileostomy, which has recently become popular in this condition.

The third case is in quite a different category, and is illustrative of some of the difficulties that may occur, not only in diagnosis, but in treatment.

A woman, aet. 8I, had had abdominal pain one year before admission. She was then thought to have had an attack of appendicitis, which had quieted down. For the past six months, she had been suffering from abdominal pain, with some difficulty in opening the bowels. This difficulty had increased in the last six to eight weeks. Four days before admission, she hegan to vomit, and the pain became much worse.

On admission, she was given an enema and a good result was obtained. When I saw her, after the result of the enema, there was some slight general abdominal distension, which was especially marked in the lower and right side of the abdomen, and although she had not vomited for two days, it looked as if there were some kind of partial obstruction present. Pelvic examination was exceedingly difficult, and I decided to give her an anaesthetic in order to make a proper bimanual examination. Under the anaesthetic, a tumour could be felt in the right iliac fossa. . It was mobile, and on the whole I was inclined to think that it was a carcinoma of the caecum. But on pelvic examination, a large tumour could be felt on the right side of the uterus, separate from it, and it was mobile and smooth; it seemed like an ovarian cyst. But the question of a carcinoma of the caecum which had come down into the pelvis was considered. Laparotomy was immediately carried out. A smooth, cystic tumour was found at the ileo-caecal angle. It was yellowish in colour and, despite her age the appearance was very like a mass of breaking-down tuberculous glands. Surrounding the tumour, the lower end of the ileum was adherent, making a sequestered loop which, in one place, was kinked and definitely obstructed. The caecum was puckered, tightly adherent to the mass, and no trace of the appendix could be seen. Under the belief that this was a mass of tuberculous glands, I incised the peritoneum to the outer side of the caecum, mobilised the gut and turned it, complete with mesentery, towards the middle line with the idea of shelling out these glands if it were possible. This very soon proved to be hopeless, as where it was adherent to the gut and despite utmost care, the mass was opened and out came a large quantity of clear, gelatinous colloid material; some of it was white and creamy; but not at all like tuberculous pus. Most of it was like white of egg.

The question of what we were dealing with was difficult. I must confess that, in the light of the pathological examination subsequently, I made a mistake. I thought we were probably dealing with a breaking-down carcinoma of a colloid nature, and I had two alternatives in front of me. The patient was not fit enough to stand a resection and anastomosis, and I was unwilling to do an anastomosis between the ileum and the transverse colon, as it would have left a blind end at the caecum, to say nothing of the exudation of fluid from the mesenteric tumour. Consequently, I thought the best thing to do would be to resect the whole mass and bring out the terminal ileum and the caecum, somewhat after the same method as in the Mikulicz operation. This procedure has been largely advocated by Devine, especially in very toxic cases of carcinoma of the caecum, although Rankin is strongly opposed to this method of treatment. I have performed it on one or two cases and it has been most successful. In view of the patient's age and her general condition, which was not good, I thought it was the method attended by the least risk. Clamps were left on both ends of the bowel until next morning when they were released. The bowel immediately began to drain itself, and her condition was good for four days, when she died rather suddenly.

Post mortem there was an extreme degree of myocarditis and slight broncho-pneumonia. The specimen removed at operation showed a cyst wall which was calcified. The appendix could not be seen or dissected out, but when the posterior wall of the cyst was cut, one got into a piece of bowel with a small lumen, which was obviously appendix. This was traced out, and although it could not be separated from the cyst wall, the terminal end appeared to open into it. We were dealing with a large mucocele of the appendix, and the histological section has confirmed this view. How the calcification is to be explained, I know not. It was this 
$t$ gave it its peculiar colour and which raised the question of tuberculosis. The condition must have been long-standing for calcification to have occurred.

This must be a rare case, and in a fairly large experience of appendicitis over many years I have not met a similar one. Evidently the appendix had perforated at the tip during the original attack, but the perforation must have been closed by the mesentery in the ileo-caecal region. Nevertheless, mucus had been poured out through the opening and had then accumulated in an adventitious sac, to which the bowel had become adherent and kinked, causing secondary obstruction. This, then, was a mistake in diagnosis. The only possible treatment, had a correct diagnosis been made, would have been drainage, which would inevitably have left a fistula.

Summary.-Three cases of disease of the ileo-caecal angle are recorded-all of them presenting a tumour, but each different in pathology.

I should like to express my thanks to Dr. Sparks of the Royal Cancer Hospital for the $\mathrm{X}$-ray which is here reproduced, and also to Dr. Bullough, Medical Officer of Health, Essex County Council, for permission to publish details of Case 3.

\section{SPENCER (BANBURY) LTD}

CONSULTANT MANUFACTURERS OF

SURGICAL, THERAPEUTIC, -MATERNITY AND ORTHOPAEDIC SUPPORTS

\section{IN DIVIDUALLY DESIGNED TO MEDICAL PRESCRIPTION FOR}

Hernia, Movable Kidney, Prolapsed Abdominal Organs, Strained Back, Spinal Abnormalities, Maternity, and

Post Operative Conditions

$$
\text { Suppliers to: }
$$

Public Health Authorities, National Health Insurance Approved Societies

Manufactory and Head Office:

spencer House, Banbury, Oxfordshire

Branches:

London, Glasgow, Edinburgh, Manchester Liverpool, Bristol, Leeds, etc.

Consultations by Appointment

\section{H E I G H M HALL, NOR W I C H}

PRIVATE MENTAL HOME for Nervous and Mental illness. All forms of treatment available. Fees from 4 gns. per week upwards, according to requirements. Vacancies occasionally exist at reduced fees on the recommendation of the patient's own physician.

Apply to Dr. J. A. SMALL

Telephone: Norwich 20080

POST-GRADUATE MEDICAL JOURNAL WAR-TIME SUBSCRIPTION FOR MEMBERS OF HIS MAJESTY'S FORCES

During the war, members of the medical profession serving with H.M. Forces may subscribe to the Journal at the annual rate of $12 \%$, post free.

This concession applies also to present Members and Associates of the Fellowship of Medicine who are now in the Forces, and the Fellowship is prepared to adjust any subscriptions paid since the outbreak of hostilities on hearing from those concerned.

(Subscribers at this special rate are not entitled to pay the lower fees, quoted to Members and Associates, for attendance at Courses of instruction.)

Fellowship of Medicine, I, Wimpole St., London, W.I. 